EPJ Web of Conferences 86,00061 (2015)

DOI: $10.1051 /$ epjconf/ 20158600061

(C) Owned by the authors, published by EDP Sciences, 2015

\title{
Comparing Experimental and Theoretical Quasifission Mass Angle Distributions
}

\author{
A. Wakhle ${ }^{1, a}$, C. Simenel ${ }^{1}$, D.J. Hinde ${ }^{1}$, M. Dasgupta ${ }^{1}$, M. Evers ${ }^{1}$, D.H. Luong ${ }^{1}$, and R. du Rietz ${ }^{1}$ \\ ${ }^{1}$ Department of Nuclear Physics, Research School of Physics and Engineering, Australian National University, Canberra, Aus- \\ tralian Capital Territory 0200, Australia
}

\begin{abstract}
We examined the ${ }^{40} \mathrm{Ca}+{ }^{238} \mathrm{U}$ reaction experimentally using the Mass-Angle Distribution (MAD) technique and within the Time Dependent Hartree Fock (TDHF) theory, using the TDHF3D code. A new, TDHF based approach has been developed to construct mass distributions as well. The results revealed that the orientation of the heavy deformed prolate nucleus plays a major role in the reaction outcome. It was found that aligned collisions lead to quasifission and short contact times of 5-10 zs, whilst anti-aligned collisions lead to longer contact times ( $>23 \mathrm{zs}$ ). TDHF accurately predicted the presence of quasifission and the average mass splits in this reaction. The influence of shell effects around ${ }^{208} \mathrm{~Pb}$ in the calculated quasifission characteristics was observed in both experiment and theory.
\end{abstract}

\section{Introduction}

Recent work on near barrier collisions of heavy nuclei is driven by a strong interest in the synthesis of superheavy elements to study the predicted 'island of stability' resulting from predicted shell closures at neutron number 184 and proton numbers between 114 to 126 [1, 2]. Forming and detecting superheavy elements is a very difficult task not only because of their minuscule (pico barn to subpico barn) formation cross sections, but also because of the reseparation of the elongated dinuclear system prior to fusion. This process is called quasifission and is a competitor to fusion. Even after fusion, the excited compound nucleus $(\mathrm{CN})$ is extremely likely to undergo fission instead of reaching the ground state of a superheavy nucleus. This process is called fusion-fission. The relationship between quasifission and fusion-fission is an important aspect of nuclear reaction dynamics. Disentangling these two processes is not always easy since their observable characteristics may overlap considerably.

Prior work has shown that the competition between fusion-fission and quasifission is affected by shell driven deformation and orientation [3, 5-8]. Shells effects encountered during the evolution of the dinuclear system were also found to affect the mass-asymmetric quasifission exit channel [9-13]. Such results provide a good testing ground for theories and models that can predict the mass and angle of fission fragments. The TimeDependent Hartree-Fock theory, which provides a selfconsistent mean-field description of the dynamics of quantum many-body systems, shows promise in describing the quasifission process and in particular these quantities [1421].

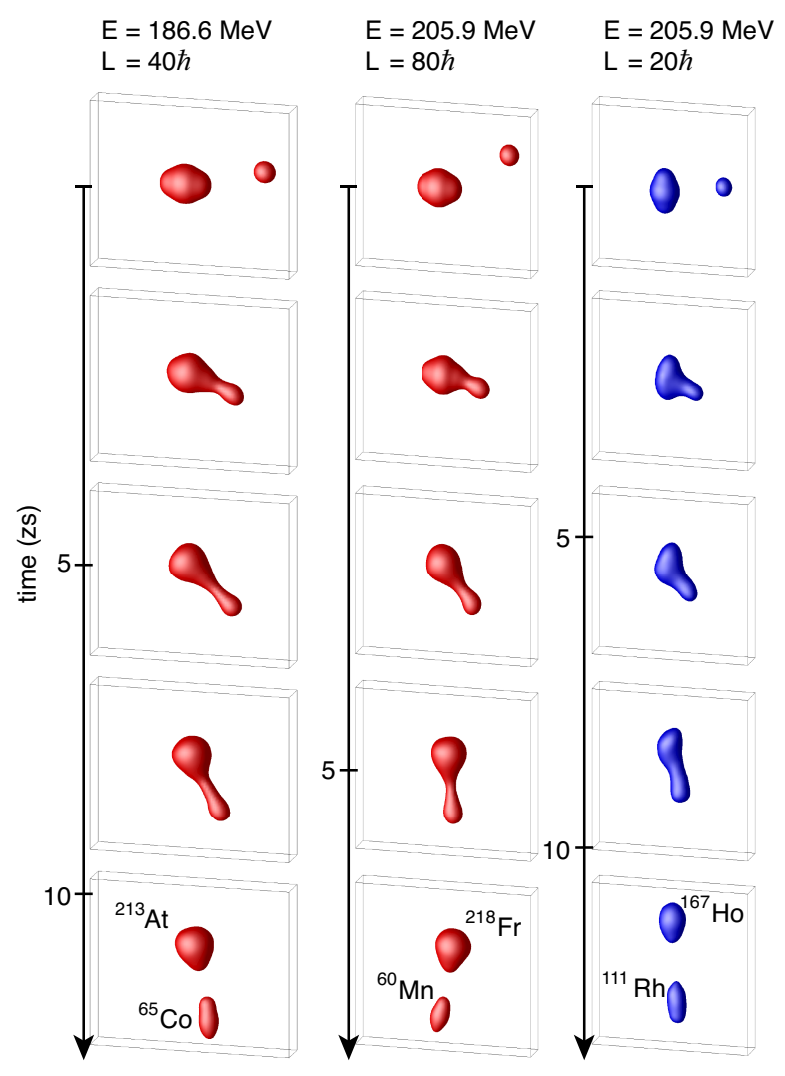

Figure 1. Isodensities at half the saturation density, i.e., $\frac{\rho_{0}}{2}=$ $0.08 \mathrm{fm}^{-3}$, in ${ }^{40} \mathrm{Ca}+{ }^{238} \mathrm{U}$ collisions. Evolutions associated to the three initial configurations, two axis (left and middle) and one equator (right), are plotted for non central collisions at energies below and above the average barrier energy. Snapshots are given at times ranging from $\mathrm{t}=0$ to reseparation, from top to bottom.

ae-mail: wakhle@nscl.msu.edu 


\section{Theoretical and Experimental Methods}

In this work we studied the ${ }^{40} \mathrm{Ca}+{ }^{238} \mathrm{U}$ reaction experimentally using the MAD technique and also using the TDHF3D code [4] to model the quasifission process. The CUBE detector system $[5,6,8]$ was used to study the binary fission fragments from the reaction and the kinematic coincidence method [6] was used to determine the massangle distributions of binary fission fragments.

The HF and TDHF calculations were both performed with the SLy4d Skyrme energy density functional [4] allowing for a fully consistent treatment of nuclear structure and reaction dynamics. In the calculations to be discussed below, the mesh size is equal to $0.8 \mathrm{fm}$ [4]. The dynamical (TDHF) calculations were performed in a half-box with $\mathrm{N}_{x}=84, \mathrm{~N}_{y}=70$ and $\mathrm{N}_{z}=28$ mesh points. This is to allow for full re-separation of the fragments and avoid spurious reflections off the hard box boundaries, before the fragments have fully separated. For all cases studied the initial separation of the nuclei is $>22.4 \mathrm{fm}$ to give the nuclei enough time in the approach phase to allow Coulomb excitation/polarisation to take place prior to contact [22].

As a compromise between computing time and adequate resolution, we chose to study three specific energies in detail. The energies chosen were as follows: one below the average Coulomb barrier where we expect the asymmetric quasifission flux to be the strongest, one just above it where we expect asymmetric quasifission and fusion fission/mass symmetric fission as well, and one well above the average barrier where quasifission is no longer expected to be the dominant exit channel and have significant overlap with fusion fission/mass symmetric fission. The impact parameter was varied by varying the initial angular momentum $L$. A range of $L$ values were studied from $L=0$ to $L=150$ in steps of $\Delta L \geq 5$. This was done to ensure that all reaction outcomes from fusion (expected at low $L$ ) to elastic scattering (expected at high $L$ ) were covered. By making this extensive series of calculations, MADs could be interpreted using TDHF calculations.

We studied two out of three extreme orientations, labelled "axis" and "equator". The axis (aligned) case corresponds to the deformation axis of ${ }^{238} \mathrm{U}$ being parallel to the $\mathrm{x}$-axis, while the equator (anti-aligned) case corresponds to it being perpendicular to the $\mathrm{x}$-axis and parallel to the $\mathrm{y}$-axis. These initial orientations are shown in figure 1 in the top most panels. In axial collisions, contact first occurs between the elongated tip of the ${ }^{238} \mathrm{U}$ nucleus and the spherical ${ }^{40} \mathrm{Ca}$ nucleus (figure 1 first column, top panel). Conversely in equatorial collisions, contact first occurs between the shortened side of the ${ }^{238} \mathrm{U}$ nucleus and the ${ }^{40} \mathrm{Ca}$ nucleus (figure 1 third column, top panel).

The calculations in this work were set for a minimum simulation time (TDHF time) of $2.3 * 10^{-20} \mathrm{~s}$, unless the system reseparated prior to this. This duration was sufficient for contact followed by a long interaction time in all cases studied. Only a few cases were studied for longer times (up to $5.4 * 10^{-20} \mathrm{~s}$ ).

The TDHF outputs give an average (expectation) value for the mass, proton and neutron numbers of each fragment. Nucleons are counted by integrating densities at the final time step over a regime where the nuclear density exceeds $0.001 \mathrm{fm}^{-3}$. The time scale is explicitly known since this is an independent variable. Based on the initial and final momentum vectors we can also determine the incoming and outgoing angles. This allows us to calculate the centre of mass angle of emission of the fragments.

\section{Interpreting Mass Angle Distributions}

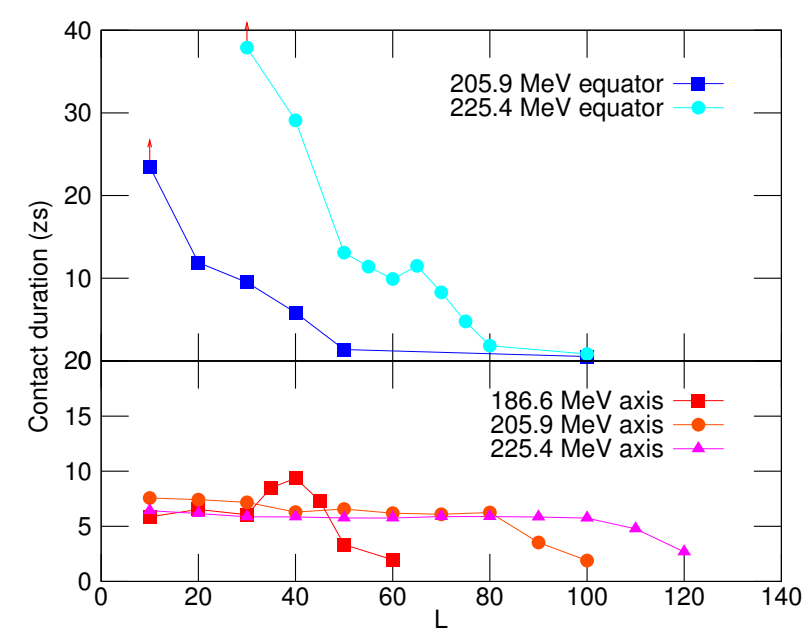

Figure 2. Contact time as a function of angular momentum L, in units of $\hbar$, for centre of mass energies corresponding to experimentally measured MADs. The points with the arrows correspond to cases where the system did not reseparate within the total time of the calculation.

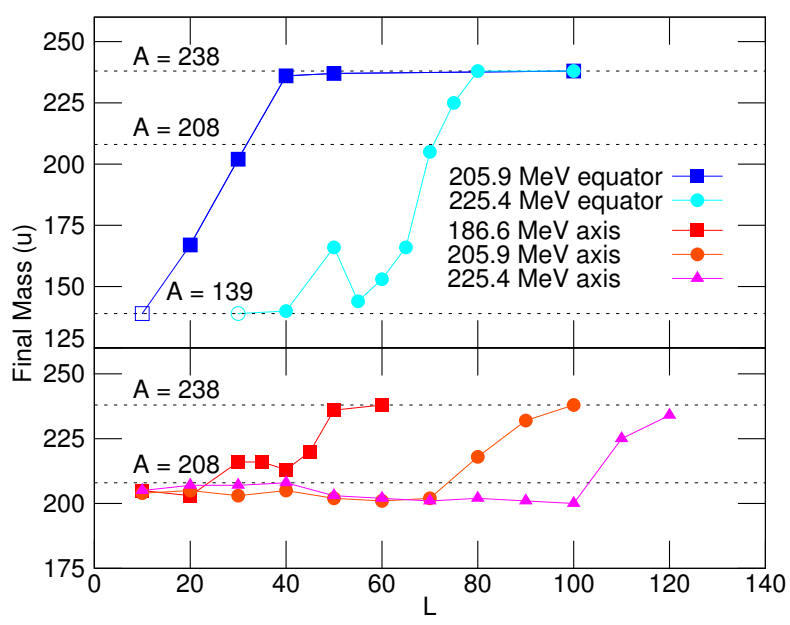

Figure 3. Final masses of the heavy fragment as a function of angular momentum $\mathrm{L}$ for centre of mass energies corresponding to experimentally measured MADs. The dotted lines correspond to $\mathrm{A}=238,208$ and 139 . The open symbols correspond to cases where the nuclei are assumed to fuse and subsequently undergo symmetric fission.

Figures 2 and 3 show the contact time and the mass of the heavy fragment as a function of L, respectively. In 
figure 3 the dotted lines represent the masses $A=208$ and $A=238$, corresponding to ${ }^{208} \mathrm{~Pb}$ and the target mass in the entrance channel, respectively. The dotted line at $A=139$ corresponds to $\frac{A_{C N}}{2}$. Overall, the total number of nucleons transferred and contact time are strongly dependent on the orientation of the ${ }^{238} \mathrm{U}$ nucleus.

For the axis orientation at $\mathrm{E}_{c . m}$. $=225.4 \mathrm{MeV}$ there is only a slight, gradual decrease in contact time with increasing $L$. The total number of nucleons transferred remains relatively constant as a function of $L$ till $L=100 \hbar$ after which it drops off. For $L<110 \hbar$ the average light/heavy split was $\mathrm{A}_{L} / \mathrm{A}_{H}=74 / 204$, consistent with mass asymmetric quasifission.

The same behaviour is seen for the axis orientation at $\mathrm{E}_{c . m .}=205.9 \mathrm{MeV}$. In this case the threshold between mass asymmetric quasifission and quasi-elastic/deep inelastic scattering is $L=80 \hbar$. For $L<80 \hbar$ the average light/heavy split remains consistent around $\mathrm{A}_{L} / \mathrm{A}_{H}=$ $75 / 203$, consistent with mass asymmetric quasifission.

Axial collisions at $\mathrm{E}_{c . m} .=186.6 \mathrm{MeV}$ only show a slight variation from this behaviour. The contact time remains relatively constant with a slight increase (30\%) between $L=30 \hbar$ and $L=45 \hbar$. Contact time drops off quickly above $L=45 \hbar$. The mass transferred remains relatively constant here as well, despite the slight increase in contact time. For $L<45 \hbar$ the average light/heavy split remains consistent around $A_{L} / A_{H}=67 / 211$, consistent with mass asymmetric quasifission.

Overall, the time-scale on which the system reseparates is between $5-10 \mathrm{zs}$, consistent with quasifission [23-25]. The mass splits are consistent around $\mathrm{A}_{L} / \mathrm{A}_{H}=$ $70 / 208$, pointing to the role of the doubly magic ${ }^{208} \mathrm{~Pb}$ shell closure in the evolution of the dinuclear system.

For the equatorial orientation at $\mathrm{E}_{c . m} .=225.4 \mathrm{MeV}$, contact time drops off sharply above $L=30 \hbar$. Below this the dinuclear system does not re-separate within the calculation time and we assume that the system forms a $\mathrm{CN}$. The total number of nucleons transferred drops off with increasing angular momentum and decreasing contact time. The same behaviour was seen for the equator orientation at $\mathrm{E}_{c . m .}=205.9 \mathrm{MeV}$ with the exception that long contact times are observed for $L<20 \hbar$ instead of $40 \hbar$ as in the previous case. It is interesting to note that the shell effects in the ${ }^{208} \mathrm{~Pb}$ region seem to have no effect in the case of equatorial collisions.

Figure 4 is a compilation of experimental MADs and masses and angles calculated by TDHF. This representation provides a clear visual comparison between the points studied in TDHF and experimental data. The pink and blue elliptical points indicate the mass and angle obtained for each TDHF calculation for a given energy and L value. The pink points correspond to the axis configuration and the blue points correspond to the equator configuration. Collisions in which the system did not reseparate are not shown since we cannot extract mass and angle values for these cases. The points are labelled with the corresponding initial angular momentum. The labels for axial collisions are shown next to the projectile-like fragments while the labels for the equatorial collisions are shown next to the target-like fragments. This is done to avoid clutter.

The size of the blue and red points in figure 4 is not indicative of the width of the mass distributions. We have used a simple model to construct mass distributions from the TDHF outputs. The mass distributions depicted in figure 4 (bottom panels) by the filled curves were generated using the TDHF-predicted mass as the centroid of a Gaussian and summing over all $L$ values using a $(2 L+1)$ distribution. The mass width $\left(\sigma_{M}\right)$ was varied from 0.02 at the entrance channel $\left(\mathrm{M}_{R}=0.144\right.$ and 0.866$)$ to 0.07 at mass symmetry $\left(\mathrm{M}_{R}=0.5\right)$ based on [25]. The heights are arbitrary and do not represent partial cross sections.

For the lowest energy $(186.6 \mathrm{MeV})$, only the axial orientation leads to contact. For $L<40 \hbar$ we see points at extreme mass asymmetry, consistent with mass asymmetric quasifission. The mass distribution calculated from TDHF (shaded pink curves) highlights the agreement with experiment. The TDHF points also show the same angular focusing as the experimental data. The experimental angular distribution, for the projectile like fragment, falls off at $\theta_{c m}=60^{\circ}$. For $L>40 \hbar$ the TDHF points depict little mass transfer and at $\theta_{c m}=60^{\circ}$ there is a transition to mass splits consistent with elastic scattering.

At the energy just above the average Coulomb barrier (205.9 MeV) both the axis and equator configurations lead to contact. For $L<80 \hbar$ the axis configuration leads to mass splits with only partial mass symmetrisation, consistent with the experimental data presented in the MAD. The drop off in the angular distribution takes place at $L=80 \hbar$ at angles beyond the coverage of the experimental setup. Hence we cannot make the same comparison here. The equator configuration populates more mass symmetric splits for $L<30 \hbar$. The corresponding modelbased mass distribution (shaded blue curve) reflects this trend and more symmetric mass splits are populated only by equatorial collisions.

At the highest energy (225.4 MeV) both the axis and equator configurations lead to contact. For $L<100 \hbar$ the axis configuration leads to mass splits consistent with mass asymmetric quasifission. At $L=100 \hbar$ the dinuclear system undergoes more than half a rotation prior to reseparating. Here too, we see a few points beyond the coverage limitations of the experimental setup and cannot use these to compare between TDHF and data. Again, only the equator configuration populates more symmetric splits. Indeed the points below $L=70 \hbar$ are consistent with more mass symmetric quasifission.

The angle of onset of elastic scattering, as predicted by TDHF, matches well with the experimental measurements. We focus on the light mass region $\left(M_{R} \approx 0.15\right)$ for the following discussion of the two highest energies where the tails of the (in)elastic peaks fall in to the angular acceptance of the CUBE detector. At $\mathrm{E}_{\text {c.m. }}=205.9 \mathrm{MeV}$ the transition to elastic scattering is at $L \geq 100 \hbar$ for axial collisions and at $L \geq 50 \hbar$ for equatorial collisions. The axial orientation populates (in)elastic scattering for angles $\theta_{c m}<90^{\circ}$. Here the equatorial orientation populates elastic scattering for angles $\theta_{c m}<125^{\circ}$, consistent with the tail 


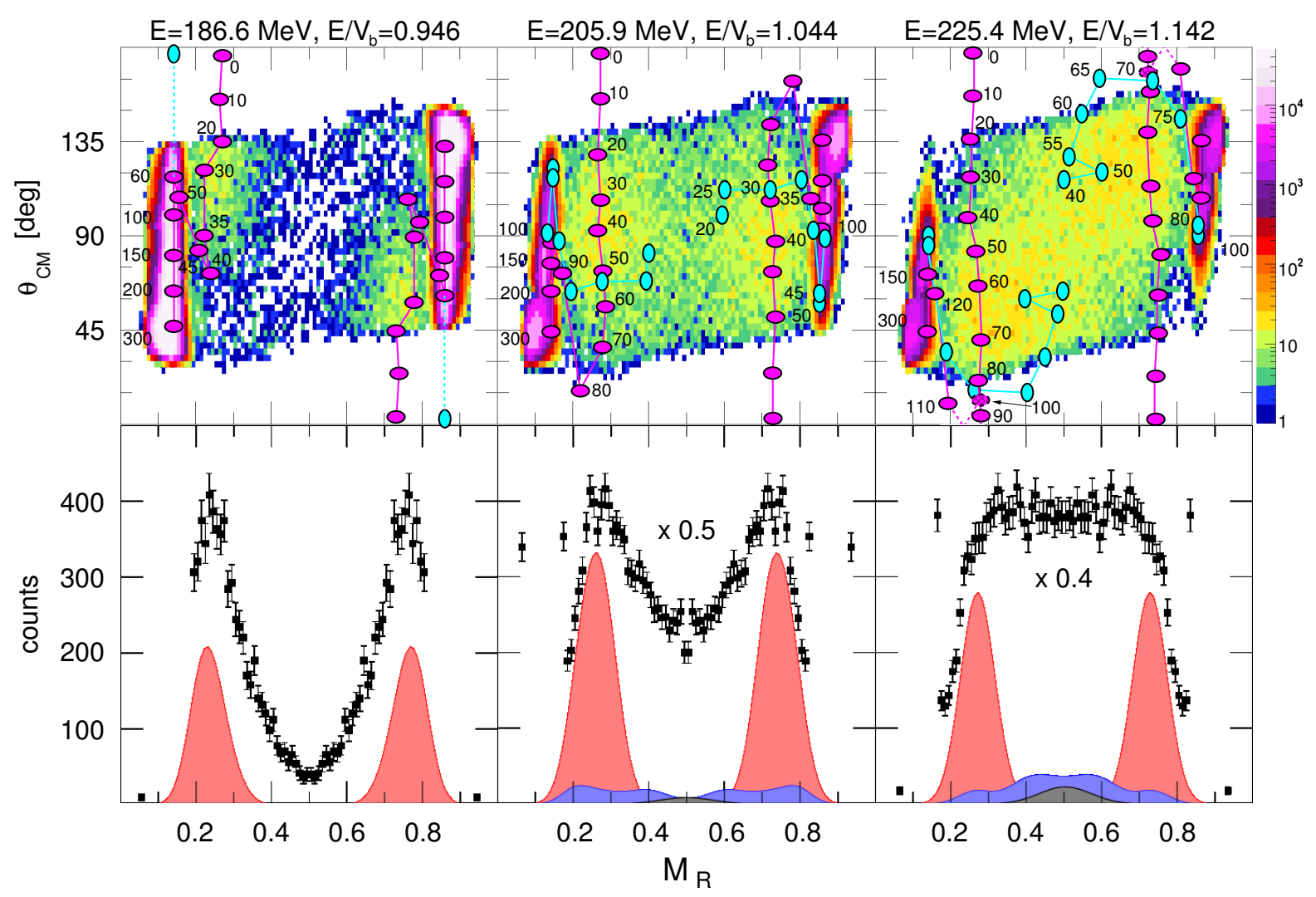

Figure 4. Top Panels: MADs for the energies studied in detail using TDHF. The elliptical points indicate the mass and angle obtained for each TDHF calculation for a given energy and $\mathrm{L}$ value. The pink points correspond to the axis configuration and the blue points correspond to the equator configuration. Bottom panel: Mass-distributions from experiment (black points) and theory (shaded curves). The curves shaded in pink correspond to the axis cases while the blue and grey curves correspond to equatorial cases that reseparate and do not reseparate within the maximum calculation time, respectively.

of the elastics seen in the MAD. Finally, at $\mathrm{E}_{\text {c.m. }}=225.4$ $\mathrm{MeV}$ it predicts (in)elastic scattering at $L \geq 150 \hbar$ for axial collisions and at $L \geq 80 \hbar$ for equatorial collisions. The axial orientation populates (in)elastic scattering for angles $\theta_{c m}<75^{\circ}$. The equatorial orientation populates (in)elastic scattering for angles $\theta_{c m}<90^{\circ}$, also consistent with the tail of the (in)elastics.

Overall, the TDHF mass splits and angular distributions show good qualitative agreement with experimental data. Calculations at intermediate orientations are needed for a quantitative comparison with experimental data.

\section{References}

[1] Yu. Ts. Oganessian et al., Phys. Rev. C 74, 044602, 2006.

[2] S. Hofmann et al., Eur. Phys. J. A 32, 251, 2007.

[3] K. Nishio et al., Phys. Rev. C 82, 044604, 2010.

[4] K.-H. Kim et al., J. Phys. G 23, 1267, 1997.

[5] D.J. Hinde et al., Phys. Rev. Lett. 101, 202701, 2008.

[6] D. J. Hinde et al., Phys. Rev. C 53, 1290, 1996.

[7] Yu. Ts. Oganessian et al., Phys. Rev. C 70, 064609, 2004.

[8] D. J. Hinde et al., Phys. Rev. Lett. 74, 1295, 1995.

[9] A. Wakhle et al., Eur. Phys. J. Web of Conferences 35, 05008, 2012.
[10] A. Wakhle et al., Phys. Rev. Lett. 113, 182502, 2014.

[11] M. G. Itkis et al., Str. Dyn. El. Mat. 66, 423, 2004.

[12] G.N. Knyazheva et al., Eur. Phys. J. Web of Conferences 63, 02014, 2013.

[13] E.M. Kozulin et al., Phys. Lett. B 686, 227, 2010.

[14] C. Golabek and C. Simenel, Phys. Rev. Lett. 103, 042701, 2009.

[15] C. Simenel, Eur. Phys. J. A 48, 152, 2012

[16] D. J. Kedziora and C. Simenel, Phys. Rev. C 81, 044613, 2010.

[17] C. Simenel et al., Eur. Phys. J. Web of Conferences 38, 09001, 2012.

[18] C. Simenel et al., J. Phys. Conf. Ser. 420, 012118, 2013.

[19] C. Simenel et al., Eur. Phys. J. Web of Conferences 63, 02001, 2013.

[20] L. Guo and T. Nakatsukasa, Eur. Phys. J. Web of Conferences 38, 09003, 2012.

[21] K. Washiyama, Contribution to FUSION14

[22] C. Simenel et al., Phys. Rev. Lett. 93, 102701, 2004

[23] J. Töke et al., Nucl. Phys. A 440, 327, 1985.

[24] W. Q. Shen et al., Phys. Rev. C 36, 115, 1987.

[25] R. du Rietz et al., Phys. Rev. Lett. 106, 052701, 2011. 